\title{
The generalized Cayley-Hamilton theorem for standard pencils
}

\section{F.R. Chang and H.C. Chen}

Department of Electrical Engineering, National Taiwan University, Taipei, Taiwan 10764, ROC

Received 27 October 1990

Revised 8 November 1991

\begin{abstract}
The Cayley-Hamilton theorem is extended from the case of one matrix to that of two matrices. The developed theorem is suitable for the standard form pencil $s E-A$. Let $\Delta(s):=\operatorname{det}(s E-A)=\sum_{r=0}^{n} a_{r} s^{r}$. Then we show that $\sum_{r=0}^{n} a_{r} A^{r} E^{n-r}=0$. Such a neat result, which is very similar to the original theorem also, is due to some nice properties of the standard pair $\{E, A\}$. For example, $E$ and $A$ commute and share the same eigenspaces.
\end{abstract}

Keywords: Cayley-Hamilton theory; singular systems; standard pencils; characteristic equations; Weierstrass forms.

\section{Introduction}

The Cayley-Hamilton theorem is very famous in linear algebra. It says that every $n \times n$ constant matrix $A$ satisfies its characteristic equation. Let

$$
\begin{aligned}
\alpha(s) & :=\operatorname{det}(s I-A) \\
& =s^{n}+\alpha_{n-1} s^{n-1}+\cdots+\alpha_{1} s+\alpha_{0} .
\end{aligned}
$$

Then

$$
\begin{aligned}
\alpha(A) & :=A^{n}+\alpha_{n-1} A^{n-1}+\cdots+\alpha_{1} A+\alpha_{0} I \\
& =0 .
\end{aligned}
$$

In this note, we will extend the theorem to the standard pencil $s E-A$ [7], where the matrix $E$ may be singular. Let

$\Delta(s):=\operatorname{det}(s E-A)=\sum_{r=0}^{n} a_{r} s^{r}$

be the characteristic polynomial of $s E-A$. Then the generalized Cayley-Hamilton theorem will show

$\Delta(E, A):=\sum_{r=0}^{n} a_{r} A^{r} E^{n-r}=0$.
The problem of the generalized Cayley-Hamilton theorem has been investigated by several researchers. In [3-6] this problem is attacked by the help of Leverrier's algorithm. Since $\{E, A\}$ is not a standard pair in their discussions, the fact of $E A \neq A E$ makes the expression pretty complicated. It is difficult to imagine why their results can be regarded as the Cayley-Hamilton theorem. [7] has developed the concept of standard pencils. Nikoukhah et al. [7] have mentioned that

$\left\{A^{n-1}, A^{n-2} E, \ldots, E^{n-1}\right\}$

spans the same subspace as

$\left\{A^{k} E^{l} \mid k, l\right.$ are nonnegative integers $\}$.

The key equation in the generalized CayleyHamilton theorem, $\Delta(E, A)=0$, is believed to be first proposed in this note.

\section{Main results}

Definition 1 [1]. Let $\hat{E}$ and $\hat{A}$ be two square constant matrices. If

$\operatorname{det}(s \hat{E}-\hat{A}) \not \equiv 0$,

then $s \hat{E}-\hat{A}$ is a regular pencil.

Definition 2 [7]. Let $s E-A$ be a regular pencil. If there exist scalars $\alpha$ and $\beta$ such that $\alpha E+\beta A=$ $I$, then $s E-A$ is a standard pencil.

Note that the characteristic polynomial of $A$ is $\operatorname{det}(s I-A)$. Here, $s I-A$ is a standard pencil since we can take $\alpha=1$ and $\beta=0$. Furthermore, any regular pencil $s \hat{E}-\hat{A}$ can be easily transformed into a standard pencil. We just let multiply $s \hat{E}-\hat{A}$ by $(\mu \hat{E}-\hat{A})^{-1}$, where $\mu$ is an arbitrary scalar satisfying

$\operatorname{det}(\mu \hat{E}-\hat{A}) \neq 0$. 
A standard pencil, $s E-A$, will be

$E:=(\mu \hat{E}-\hat{A}){ }^{1} \hat{E}$,

and

$A:=(\mu \hat{E}-\hat{A})^{-1} \hat{A}$.

If we choose $\alpha=\mu$ and $\beta=-1$, then

$\mu E-A=I$.

Hence, it is clear that $s E-A$ is indeed a standard pencil. In the following, we assume that $\{E, A\}$ is an $n \times n$ standard pair satisfying (3) in which $E$ may be singular. First of all, we will construct the Weierstrass form [1] of $s E-A$. Let $U$ be the modal matrix of $E$. Then $E$ can be similarly transformed into its Jordan form,

$U^{-1} E U=\left[\begin{array}{cc}E_{\mathrm{s}} & 0 \\ 0 & E_{\mathrm{f}}\end{array}\right]=: \bar{E}$,

where $E_{\mathrm{s}}$ is an $r \times r$ nonsingular matrix and $E_{\mathrm{f}}$ is an $(n-r) \times(n-r)$ nilpotent matrix. From (3), $A=\mu E-I$, and hence

$$
\begin{aligned}
U^{-1} A U & =\left[\begin{array}{cc}
\mu E_{\mathrm{s}}-I & 0 \\
0 & \mu E_{\mathrm{f}}-I
\end{array}\right] \\
& =\left[\begin{array}{cc}
A_{\mathrm{s}} & 0 \\
0 & A_{\mathrm{f}}
\end{array}\right]:=\bar{A},
\end{aligned}
$$

where the sizes of $A_{\mathrm{s}}$ and $A_{\mathrm{f}}$ are the same as those of $E_{\mathrm{s}}$ and $E_{\mathrm{f}}$ respectively. The standard pencil thus can be transformed into

$$
\begin{aligned}
U^{-1}(s E-A) U & =(s \bar{E}-\bar{A}) \\
& =\left[\begin{array}{cc}
s E_{\mathrm{s}}-A_{\mathrm{s}} & 0 \\
0 & s E_{\mathrm{f}}-A_{\mathrm{f}}
\end{array}\right]
\end{aligned}
$$

in which $s E_{\mathrm{s}}-A_{\mathrm{s}}$ and $s E_{\mathrm{f}}-A_{\mathrm{f}}$ represent the slow and fast part of the Weierstrass form.

Once we have (6), the characteristic polynomial of $s E-A$ will be shown to be the same as that of $s E_{\mathrm{s}}-A_{\mathrm{s}}$. Let

$\operatorname{det}\left(s E_{\mathrm{s}}-A_{\mathrm{s}}\right)=a_{r} s^{r}+a_{r-1} s^{r-1}+\cdots+a_{1} s+a_{0}$.

Then $a_{r}=\operatorname{det}\left(E_{\mathrm{s}}\right) \neq 0$. Also,

$\operatorname{det}\left(s E_{\mathrm{f}}-A_{\mathrm{f}}\right)=\operatorname{det}\left(s E_{\mathrm{f}}-\mu E_{\mathrm{f}}+I\right)=1$, since $E_{\mathrm{f}}$ is a nilpotent matrix in Jordan form. So,

$$
\begin{aligned}
\operatorname{det}(s E-A)= & \operatorname{det}(s \bar{E}-\bar{A}) \\
= & \operatorname{det}\left(s E_{\checkmark}-A_{s}\right) \\
= & a_{r} s^{r}+a_{r-1} s^{r-1}+\cdots+a_{1} s+a_{0} \\
= & a_{n} s^{n}+a_{n-1} s^{n-1}+\cdots \\
& +a_{r} s^{r}+\cdots+a_{1} s+a_{0}
\end{aligned}
$$

where $a_{n}=a_{n-1}=\cdots=a_{r+1}=0$, and $a_{r} \neq 0$.

Some nice properties, such as $E$ and $A$ commuting, of a standard pair will be investigated.

Lemma 1. (i) $E A=A E$.

(ii) $\bar{E} \bar{A}=\overline{A E}$.

(iii) $E_{\mathrm{s}} A_{\mathrm{s}}=A_{\mathrm{s}} E_{\mathrm{s}}$ and $E_{\mathrm{f}} A_{\mathrm{f}}=A_{\mathrm{f}} E_{\mathrm{f}}$.

(iv) for any nonnegative integers $l, k$,

$E^{k} A^{l}=U\left(\bar{E}^{k} \bar{A}^{\prime}\right) U^{-1}=U\left(\vec{A}^{k} \bar{E}^{k}\right) U^{-1}$.

Proof. (i) By (3), $A=\mu E-I$. So,

$E A=E(\mu E-I)=(\mu E-I) E=A E$.

(ii) By (4) and (5),

$$
\begin{aligned}
\bar{E} \bar{A} & =\left(U^{-1} E U\right)\left(U^{-1} A U\right)=U^{-1} E A U \\
& =U^{-1} A E U=\left(U^{-1} A U\right)\left(U^{-1} E U\right)=\overline{A E} .
\end{aligned}
$$

(iii) It is clear by the result of (ii) as well as (4) and (5).

(iv) It is clear by noting that $U^{-1} U=I, E=$ $U \bar{E} U^{-1}, A=U \bar{A} U^{-1}$, and $\bar{E} \bar{A}=\overline{A E}$.

The Cayley-Hamilton theorem of slow part pencil, $s E_{s}-A_{s}$, is described by the next lemma.

Lemma 2. If

$\operatorname{det}\left(s E_{\mathrm{s}}-A_{\mathrm{s}}\right)=\sum_{i=0}^{r} a_{i} s^{i}$,

then

$\Delta\left(E_{s}, A_{s}\right):=\sum_{i=0}^{r} a_{i} A_{\mathrm{s}}^{i} E^{r-i}=0$.

Proof. We have

$$
\begin{gathered}
\operatorname{det}\left(s E_{\mathrm{s}}-A_{\mathrm{s}}\right)=\operatorname{det}\left(E_{\mathrm{s}}\right) \operatorname{det}\left(s I-E_{\mathrm{s}}^{-1} A_{\mathrm{s}}\right) \\
\quad=a_{r}\left(s^{r}+\tilde{a}_{r-1} s^{r-1}+\cdots+\tilde{a}_{1} s+\tilde{a}_{0}\right)
\end{gathered}
$$


where $a_{r}=\operatorname{det}\left(E_{\mathrm{s}}\right) \neq 0$ and $\tilde{a}_{i}=a_{i} / a_{r}$ for $i=$ $1,2, \ldots, r-1$

By applying the Cayley-Hamilton theorem to $E_{\mathrm{s}}^{-1} A_{\mathrm{s}}$, we have

$$
\begin{aligned}
& \left(E_{\mathrm{s}}^{-1} A_{\mathrm{s}}\right)^{r}+\tilde{a}_{r-1}\left(E_{\mathrm{s}}^{-1} A_{\mathrm{s}}\right)^{r-1}+\cdots \\
& \quad+\tilde{a}_{1}\left(E_{\mathrm{s}}^{-1} A_{\mathrm{s}}\right)+\tilde{a}_{0} I=0 .
\end{aligned}
$$

If we left multiply the above equation with $a_{r} E_{\mathrm{s}}^{r}$ and use the result of Lemma 1(iii), $E_{s} A_{s}=$ $A_{s} E_{s}$, we have

$$
\begin{aligned}
& a_{r} A_{s}^{r}+a_{r-1} A_{s}^{r-1} E_{s}+\cdots \\
& \quad+a_{1} A_{s} E_{s}^{r-1}+a_{0} E_{s}^{r}=0 .
\end{aligned}
$$

Now, the main theorem of the generalized Cayley-Hamilton theorem for the standard pencil is ready to be proved.

Theorem 1. Generalized Cayley-Hamilton Theorem. The standard pair $\{E, A\}$ satisfies its own characteristic equation; that is, $\Delta(E, A)=0$, where $\Delta(s)=\operatorname{det}(s E-A)$ is given in (7).

\section{Proof.}

$$
\begin{aligned}
& \Delta(E, A) \\
& :=a_{n} A^{n}+a_{n-1} A^{n-1} E+\cdots \\
& +a_{r} A^{r} E^{n-r}+\cdots+a_{1} A E^{n-1}+a_{0} E^{n} \\
& =\left(a_{r} A^{r}+a_{r-1} A^{r-1} E+\cdots\right. \\
& \left.+a_{1} A E^{r-1}+a_{0} E^{r}\right) E^{n-r} \\
& =\left\{U \left(a_{r} \bar{A}^{r}+a_{r-1} \bar{A}^{r-1} \bar{E}+\cdots\right.\right. \\
& \left.\left.+a_{1} \overline{A E}^{r-1}+a_{0} \bar{E}^{r}\right) U^{-1}\right\} \\
& \cdot\left\{U \bar{E}^{n-r} U^{-1}\right\} \\
& =U\left\{a_{r}\left[\begin{array}{cc}
A_{\mathrm{s}}^{r} & 0 \\
0 & A_{\mathrm{f}}^{r}
\end{array}\right]\right. \\
& +a_{r-1}\left[\begin{array}{cc}
A_{\mathrm{s}}^{r-1} & 0 \\
0 & A_{\mathrm{f}}^{r-1}
\end{array}\right]\left[\begin{array}{cc}
E_{\mathrm{s}} & 0 \\
0 & E_{\mathrm{f}}
\end{array}\right]+\cdots \\
& +a_{1}\left[\begin{array}{cc}
A_{\mathrm{s}} & 0 \\
0 & A_{\mathrm{f}}
\end{array}\right]\left[\begin{array}{cc}
E_{\mathrm{s}}^{r-1} & 0 \\
0 & E_{\mathrm{f}}^{r-1}
\end{array}\right]
\end{aligned}
$$

$$
\begin{aligned}
& \left.+a_{0}\left[\begin{array}{cc}
E_{\mathrm{s}}^{r} & 0 \\
0 & E_{\mathrm{f}}^{r}
\end{array}\right]\right\}\left[\begin{array}{cc}
E_{\mathrm{s}}^{n-r} & 0 \\
0 & E_{\mathrm{f}}^{n-r}
\end{array}\right] U^{-1} \\
= & U\left[\begin{array}{cc}
\Delta\left(E_{\mathrm{s}}, A_{\mathrm{s}}\right) & 0 \\
0 & \Delta\left(E_{\mathrm{f}}, A_{\mathrm{f}}\right)
\end{array}\right]\left[\begin{array}{cc}
E_{\mathrm{s}}^{n-r} & 0 \\
0 & 0
\end{array}\right] U^{-1} \\
= & U\left[\begin{array}{cc}
0 & 0 \\
0 & \Delta\left(E_{\mathrm{f}}, A_{\mathrm{f}}\right)
\end{array}\right]\left[\begin{array}{cc}
E_{\mathrm{s}}^{n-r} & 0 \\
0 & 0
\end{array}\right] U^{-1} \\
= & 0 .
\end{aligned}
$$

During the derivation, we have used $\Delta\left(E_{\mathrm{s}}, A_{\mathrm{s}}\right)$ $=0($ Lemma 2$)$ and $E_{\mathrm{f}}^{n-r}=0\left(E_{f}\right.$ is an $(n-r) \times$ $(n-r)$ nilpotent matrix).

\section{Conclusion}

We have developed the generalized CayleyHamilton theorem for standard pencils. Some nice properties of the standard pair $\{E, A\}$, such as $E$ and $A$ commuting, the eigenspaces of $E$ and $A$ being the same, etc. were used to simplify the mathematical expressions. The final result is very neat. One possible contribution of the generalized Cayley-Hamilton theorem seems to be in analyzing and designing solvable singular systems. It is well known that in order to guarantee the existence and the uniqueness of the solution of

$\hat{E} \dot{x}=\hat{A x}+\hat{B} u$,

$s \hat{E}-\hat{A}$ must be a regular pencil [8]. As mentioned in (1) and (2), if we left multiply ( $\mu \hat{E}-$ $\hat{A})^{-1}$ on both sides of the singular system equation, then we have

$E \dot{x}=A x+B u$,

where

$E=(\mu \hat{E}-\hat{A})^{-1} \hat{E}$

$A=(\mu \hat{E}-\hat{A})^{-1} \hat{A}$,

and

$B=(\mu \hat{E}-\hat{A})^{-1} \hat{B}$.

Now $s E-A$ is a standard pencil, and hence the generalized Cayley-Hamilton theorem can be applied. 


\section{References}

[1] F.R. Gantmacher, The Theory of Matrices, Vol. 2 (Chelsea, New York, 1974) p. 25.

[2] T. Kailath, Linear Systems (Prentice-Hall, Englewood Cliffs, NJ, 1980) p. 395.

[3] F.L. Lewis, Further remarks on the Cayley-Hamilton theorem and Leverrier's method for the matrix pencil $(s E-$ A), IEEE TRans. Automat. Control 31 (1986) 869-870.

[4] F.L. Lewis, Cayley-Hamilton theorem and Fadeev's method for the matrix pencil ( $s E-A$ ), Proc. 22nd IEEE Conf. Decision Control (1982) 1282-1288.
[5] B.G. Mertzios, Leverrier's algorithm for singular systems, IEEE Trans. Automat. Control 29 (1984) 652-653.

[6] B.G. Mertzios and M.A. Christodoulou, On the generalized Cayley-Hamilton theorem, IEEE Trans. Automat. Control 31 (1986) 156-157.

[7] R. Nikoukhah, A.S. Willsky and B.C. Levy, Boundary-value descriptor systems: well-posedness, reachability and observability, Internat. J. Control 46 (1987) 1715-1737.

[8] G.C. Verghese, B.C. Levy and T. Kailath, A generalized state-space for singular systems, IEEE Trans. Automat. Control 26 (1981) 811-831. 